\title{
OPTICAL OBSERVATIONS OF FY PERSEI
}

\author{
AKIRA OKAZAKI \\ Department of Science Education, Gunma University, Maebashi, Japan
}

\begin{abstract}
The results are presented of multi-channel photometry and spectroscopy of the suspected nova-like variable FY Per. These results suggest that FY Per may be a Herbig $\mathrm{Ae} / \mathrm{Be}$ star rather than a nova-like variable.
\end{abstract}

\section{Introduction}

The light variability of FY Persei was first detected by Morgenroth (1936). Richter (1964) examined photographic data of FY Per from 1935 to 1963, and found that the light of FY Per varies irregularly between 11.2 and 14.7 mag. Subsequently, Shugarov (1980) found tentative evidence for periodicity on a few hour time scale from his $U B V$ photoelectric observations. $\mathrm{He}$ suggested that FY Per may be a nova-like variable. Ritter (1990) also listed this star in his catalogue of cataclysmic binaries.

The nature of FY Per, however, still remains an open question, because neither spectroscopic studies nor further photometric ones of FY Per have been published.

\section{The Observations}

\subsection{Multi-channel Photometry}

We observed FY Per at the Dodaira Station of the National Astronomical Observatory of Japan (NAOJ), with the 0.91-m telescope equipped with the eight-channel polarimeter (ch.1: $\lambda_{\text {eff }}=360 \mathrm{~nm}$, ch.2: $410 \mathrm{~nm}$, ch. $3: 460 \mathrm{~nm}$, ch.4: $530 \mathrm{~nm}$, ch.5: $650 \mathrm{~nm}$, ch.6: $700 \mathrm{~nm}$, ch.7: $760 \mathrm{~nm}$, ch.8: $880 \mathrm{~nm}$. see Kikuchi, 1988), on 13 nights from October 1989 to February 1991. An integration time in each observation was $36 \mathrm{~s}$. We chose two nearby stars as the comparision stars.

We obtained $\sim 2400$ individual observations in each channel (The data in ch. 8 have not been used in this study because of their poor $S / N$ ). The observed light curve of FY Per (in ch.4) shows irregular variation with an amplitude of $\sim 0.5 \mathrm{mag}$, as presented in Fig. 1, where each cross is a normal point consisting of 30 individuals (the estimated error of each normal is 0.01-0.02 mag). It is noticed that FY Per occasionally exhibit rapid light variation, as mentioned by Richter (1964). For example, on January 24,1990 , FY Per became fainter by 0.15 mag for a few hours.

We also found that the color of FY Per becomes redder when it dims as far as the observed magnitude range. For example, FY Per moves almost 


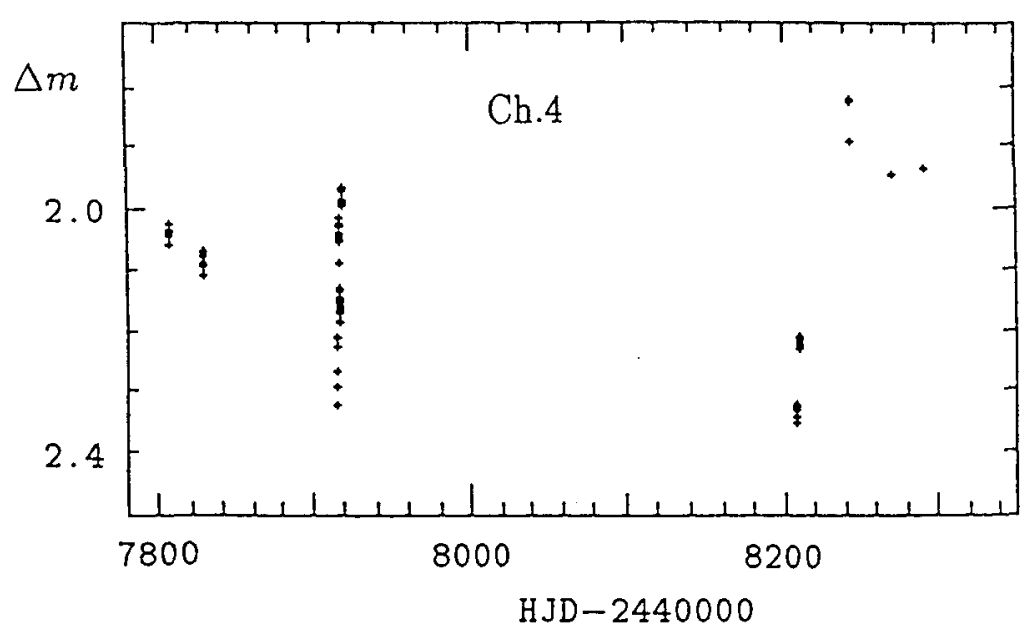

Fig. 1. The observed light curve of FY Per in ch. 4.

along the line represented by $\Delta m_{4} / \Delta\left(m_{3}-m_{4}\right) \approx 10$ in the color-magnitude diagram.

\subsection{SPECTROSCOPY}

We obtained three spectrograms with a dispersion of $\sim 220 \AA \mathrm{mm}^{-1}$ on baked IIa-O emulsion with exposure times of $50-65 \mathrm{~min}$ on February 14, 1989, using the Cassegrain image-tube spectrograph of the 1.88-m telescope at the Okayama Astrophysical Observatory of NAOJ.

We found that FY Per shows a rather narrow $\mathrm{H} \alpha$ emission line (corresponding to a velocity dispersion of $150-200 \mathrm{~km} \mathrm{~s}^{-1}$ ), though other members of Balmer series are absorption lines which are considered to originate from an early-type star.

\section{Discussion}

Shugarov (1980) found tentative evidence for periodicity on a few hour time scale in the light of FY Per on the basis of his 23 observations. He deduced several periodicity candidates such as $0.0702 \mathrm{~d}$ and $0.4407 \mathrm{~d}$. Then, we made a period analysis for all the $\sim 2400$ individual observations (the combined light of Ch.2, 3 and 4) using the PDM method (Stellingwerf, 1978). We found, however, no definite evidence for such short periodicity.

As mentioned above, (1) FY Per shows irregular light variation, (2) the color of FY Per becomes redder when it dims, and (3) FY Per has a rather narrow $\mathrm{H} \alpha$ emission line which is superimposed on an early-type star spectrum. It is also noticed that FY Per is located near by the dark nebula 
complex B20.

These observational facts suggest that FY Per may be a Herbig Ae/Be star rather than a nova-like variable.

\section{Acknowledgements}

We would like to thank Dr. S. Kikuchi for helpful discussions. We are also grateful to Mr. T. Kitazume and the staffs of the Dodaira Station and the Okayama Astrophysical Observatory of NAOJ for their assistance with the observations.

\section{References}

Kikuchi, S.: 1988, Tokyo Astron. Bull., second ser., 3267.

Morgenroth, O.: 1936, Astron. Nachr., 261, 261.

Richter, G.: 1964, Mitt. Veränderl. Sterne, 2, No.4, 79.

Ritter, H.: 1990, Astronomy and Astrophysics, Supplement Series, 85, 1179.

Shugarov, S. Yu.: 1980, Astron. Tsirk., No. 1119, 3.

Stellingwerf, R. F.: 1978, Astrophysical Journal, 224, 953. 